\title{
The role of pivots in exolingual communication
}

\section{[Le role du pivot dans la communication exolingue]}

\author{
Katarina Chovancova - Carolina Haro Guerrero
}

DOI: 10.18355/XL.2020.13.01.05

\begin{abstract}
The paper deals with exolingual trilogues between native and non native speakers of Slovak and French, characterized by a specific distribution of communicative tasks. The presence of a mediator, i. e. a communicating subject who facilitates the exchange between the (two) other interlocutors not having a common language available that would be mastered with an equal competence by all, is marked by repetitive occurrences of signals of refomulation as a central discursive strategy ensuring the success of the communication. Basing their analysis on authentic linguistic material gathered for the needs of empirical research on exolingual and multicode communication, the authors identify pertinent features of communicative behaviour of the mediator.
\end{abstract}

Key words: language, linguistics, mediation, reformulation, exolingual

\section{Résumé}

L'article porte sur les trilogues exolingues entre locuteurs natifs et non natifs du slovaque et $\mathrm{du}$ français caractérisés par une répartition particulière des rôles communicatifs. La présence du pivot, sujet communiquant facilitant l'échange entre les (deux) autres interlocuteurs ne disposant pas de langue commune qui soit également bien maîtrisée de tous, entraîne les marques récurrentes de la reformulation, stratégie discursive centrale pour assurer la réussite communicative. À la base du matériau linguistique authentique, rassemblé par les auteures pour les besoins de la recherche empirique sur la communication exolingue et multicode, l'identification des traits saillants du comportement communicatif du pivot est réalisée.

Mots-clefs : langue, linguistique, médiation, reformulation, exolingue

\section{Introduction}

La communication exolingue est définie comme toute communication dans laquelle se mettent en présence des répertoires langagiers individuels hétérogènes du point de vue des niveaux de compétences communicatives des sujets parlants. Ce type de communication peut être observée dans les contextes d'enseignement les plus divers, mais également dans toutes les situations communicatives de tous les jours marquées par la co-présence des locuteurs natifs et non natifs d'une langue. Les contextes communicatifs de ce genre particulier sont fréquents là où il s'agit d'une mobilité d'études, de travail et de recherche, ainsi que de toute sorte de mouvement migratoire des individus à travers des communautés linguistiques. Les défis de la communication exolingue consistent en des obstacles communicatifs découlant de la maîtrise inégale de la langue qui est choisie pour communiquer et en la nécessité de se forger des solutions à des manques des ressources et des compétences. Le plus souvent, la recherche de ces solutions amène les locuteurs à se forger des stratégies pragmatiques palliatives spécifiques. Une autre issue pour les situations de communication exolingues peut consister à avoir recours à l'aide d'un locuteur-médiateur agissant de pivot qui facilite l'échange autrement menacé par un risque élevé d'incompréhension. L'étude des communications exolingues à pivot fait objet de cette recherche. 


\section{Cadre théorique}

Pour défnir le cadre théorique de cette étude il est nécessaire d'introduire deux concepts qui y sont centraux : la communication exolingue et la communication à pivot (médieé).

\section{La communication exolingue}

La communication exolingue «s'établit entre locuteurs n'ayant pas une L1 commune " (Porquier 1979: 50). Elle s'oppose à la communication endolingue, menée par les locuteurs ayant une même langue maternelle. En d'autres ter,es, "la communication exolingue est celle qui s'établit [...] par des moyens autres qu'une langue maternelle éventuellement commune aux participants » (Porquier $1984: 18$ ). Selon Bernier (2007), à la suite de Bange (1992), l'interaction exolingue « survient entre au moins un locuter natif de la langue utilisée comme véhicule de la communication en cours et un alloglotte, locuteur non natif de cette langue, appartenant généralement à un groupe socioculturel étranger ». Certains associent le caractère exolingue d'un échange à la différence des répertoires des participants (De Pietro 1988) où à l'inégalité des compétences linguistiques des interlocuteurs par rapport à la langue de la communication en cours (Dausendschön-Gay $2003: 43$ ). Une non identité des langues de communication disponibles aux locuteurs et l'existence des niveaux inégaux des compétences communicatives des interlocuteurs dans une langue peuvent se combiner. Comme le constate Porquier $(2003: 51)$ : « Si l'on accepte de caractériser la communication exolingue par une asymétrie entre les répertoires ou les compétences des participants, celle-ci peut concerner la langue ou les langues (langue non maternelle de l'un au moins des participants) de l'échange ou bien le degré d'expertise (spécialiste/non spécialiste, médecin/patient, etc.) dans le domaine de l'échange, ou les deux. Dans ce dernier cas, l'asymétrie peut être double, et croisée ». Le plus souvent, le terme de communication exolingue est utilisé en association à la communication entre un natif et un non natif (un alloglotte) ou entre des locuteurs bilingues. Le fait de ne pas appartenir à la même commmunauté linguistique y est essentiel :

«La communication est exolingue lorsque les divergences entre les répertoires linguistiques respectifs des interlocuteurs apparaissent comme constitutives du fonctionnement de l'interaction, c'est-à-dire lorsque le recours à des procédés d'ajustement réciproque, d'auto/hétérofacilitation, etc, devient un trait saillant de la communication. C'est le cas généralement des interactions entre natif et alloglotte, entre médecin et patient (Lacoste, 1980), entre parents et enfant (Snow et Ferguson [eds.], 1977), etc, qui, d'un point de vue linguistique, et souvent sociologique, sont asymétriques. La communication devient endolingue, au contraire, lorsque les divergences codiques ne représentent plus une donnée pertinente dans la gestion du discours, autrement dit lorsqu'elles ne sont plus perçues comme significatives par les participants à l'événement langagier. » (De Pietro 1988 : 71)

Selon De Pietro, le caractère exolingue vs. endolingue, tout comme le caractère unilingue $v s$. bilingue, sont des paramètres définitoires de la situation de contact de langues. L'auteur propose un ensemble de quatre situations prototypiques: a) l'endolingue / unilingue, b) l'exolingue / unilingue, c) l'exolingue / bilingue et d) l'endolingue / bilingue. Rosen et Reinhardt (2003 : 93) rajoutent le paramètre d'expertise, en opposant les locuteurs experts aux locuteurs non experts. L'expert est caractérisé comme " la personne ressource qui remplit les fonctions d'informateur, de réparateur et d'évaluateur » (Hudelot, Vasseur 1997 : 110, in Rosen, Reinhardt, 2003 :

XLinguae, Volume 13 Issue 1, January 2020, ISSN 1337-8384, eISSN 2453-711X 
9). Selon le modèle de communication proposé par Kerbrat-Orecchioni (2002), le caractère exolingue résiderait également en la non identité des bagages cognitifs des interactants n'étant jamais idéalement monolingues. Dans cette conception large, l'asymétrie est vue comme inhérente à toute interaction.

Nous proposons d'attirer l'attention, à l'intérieur du concept de communication exolingue, sur les communications qui ont lieu entre $X$, locuteur natif (ou assimilé) de langue $\mathrm{A}$, et $\mathrm{Y}$, locuteur avec compétence zéro en langue A. Il s'agit d'une asymétrie maximale; on assiste à la communication dans une langue presque ou complètement étrangère à l'un des interlocuteurs. Une telle situation peut se produire, par exemple, lors d'un voyage dans un pays dont on ne parle pas la langue, étant obligé de se faire comprendre (voir aussi l'étude de Behrent 2008 sur les interactions interalloglottes). Dans ce cas-là, la question est celle des solutions à adopter en vue de la réussite communicationnelle face à non identité des codes utilisés par les locuteurs. La possibilità de recourir à la médiation par un intermérdiaire humain soulève un intérêt spécifique.

\section{la communication à pivot}

La communication à pivot se déploie entre trois locuteurs au minimum dont l'un adopte le rôle spécifique de pivot (médiateur) de l'échange entre les deux autres. Le cadre participatif, traditionnellement formé de deux pôles, est ici élargi d'un troisième membre, actif dans l'espace intermédiaire entre ces deux instances énonciatrices de base. Or, le terme de pivot désigne une personne physique dotée d'un bagage cognitif et d'un comportement communicatif propres, tel un messager, un traducteur, un interprète, un instituteur, un rédacteur etc. (cf. Kerbrat-Orecchioni, $2002: 25$ ).

Le caractère pivoté (médié) de la communication est associé, dans notre conception, uniquement au concept de la médiation humaine et ne se confond pas avec le caractère médiatisé ni médiatique. Il n'est pas lié non plus à la présence ou à l'absence du support technique véhiculant le message (caractère indirect ou direct). La coprésence physique des locuteurs n'a pas de pertinence pour la communication médiée. Dans ce processus à deux étapes, il y a d'abord le transfert de l'information entre le locuteur 1 (le destinateur originel du message) et le locuteur 2 (le pivot). Ensuite, il y a le transfert entre le locuteur 2 (le pivot) et le locuteur 3 (le destinataire final). Dans les deux phases, il s'agit d'un transfert accompagné de back-channelling, même si le retour d'information de la part du locuteur 2 vers le locuteur 1 n'est pas nécessairement parfait, surtout s'il s'agit d'une médiation simultanée. La communication à trois n'est pas toujours une communication à pivot. Elle peut se réaliser au quotidien, sous forme des rencontres conversationnelles ordinaires entre trois personnes, sans que l'une se différencie des autres par son rôle communicatif (cf. Plantin et Kerbrat-Orecchioni, 1995).

L'essentiel du rôle de pivot tient à la modification du message transféré sous l'influence de l'ensemble des attributs personnels, intellectuels et affectifs du locuteur concret et sous l'influence des autres composantes du contexte extralinguistique, dont le temps et, secondairement, l'espace. Le rôle de pivot consiste à une reproduction du contenu informationnel avec un degré variable de sq modification, parfois même à un recodage complet vers une autre langue naturelle.

\section{Cadre théorique et méthodologie}

La recherche porte sur les conversations quotidiennes où les participants sont dotés de divers niveaux de compétences communicatives dans les langues différentes, mais ne disposent pas tous d'une langue de communication commune. L'analyse empirique est réalisée à partir d'un ensemble de données recueillies sous forme de corpus de conversations orales. Le corpus consiste d'enregistrements audio originels des événements communicatifs (conversations) dans la durée totale de 5 heures. Il s'agit 
des communications orales, privées, non officielles, improvisées, impliquant 3 ou 4 participants. La langue maternelle des participants n'est pas la même. Certains ne maîtrisent pas du tout celle de l'autre. Ainsi, il y a des locuteurs natifs slovaques qui ne parlent pas le français; des locuteurs natifs français sans connaissance du slovaque ; enfin, il y a des Slovaques et des Français qui n'ont qu'une compétence partielle dans la langue de l'autre.

Le corpus de travail est construit en collaboration avec l'équipe du Corpus national du slovaque, plus spécifiquement le Corpus oral du slovaque, en ce qui concerne les paramètres qualitatifs exigés du format des enregistrements, au système de transcription et d'annotation du corpus. À ces exigences de base s'ajoutent d'autres, liées à l'annotation des phénomènes pragmatiques et communicatifs typiques de la situation de communication exolingue.

Les productions linguistiques des participants sont analysées du point de vue de la présence des phénomènes choisis liés au contact des langues. Ces phénomènes sont évalués en rapport aux paramètres sociologiques des participants. Étant donné le caractère dialogique du matériel recueilli, il est nécessaire, lors de son analyse, d'avoir recours aux méthodes de l'analyse transversale et de l'analyse longitudinale des interactions verbales. Il est question d'identifier les traces linguistiques des stratégies communicatives mises en place, de leur catégorisation et de leur interprétation. Il s'avère intéressant, également, d'observer la signalisation métalinguistique des diverses stratégies communicatives.

Les stratégies communicatives adoptées par les participants seront envisagées sous perspective de leur efficacité, c'est-à-dire de leur potentiel en vue d'assurer la réussite de l'échange et d'éliminer le risque des malentendus et des incompréhensions.

La communication exolingue observée dans le corpus de travail est caractérisée par la prise en compte, de la part des participants qui ont une compétence limitée dans la langue de l'autre, du fait qu'ils communiquent dans une langue acquise (non maternelle). Cette caractéristique - la conscience de la disparité chez les locuteurs (cf. Cappellini 2017) - peut être considérée comme foncière dans toute interaction exolingue. Quant au choix des stratégies de communication, une approche coopérative est présente chez tous les participants, ainsi qu'une tolérance plus élevée vis-à-vis des écarts de la norme linguistique et le soutien de la part des participants plus compétents envers ceux qui le sont moins se matérialisant dans des stratégies d'hétérofacilitation. Le recours à des stratégies spécifiques d'ajustement relatives au choix des moyens lexicaux (la recherche du mot juste liée à la répétition et à la reprise, l'emploi des néologismes, la traduction, la paraphrase, les descriptions etc.) est aussi présent. Les participants essaient de prévenir les problèmes communicatifs et l'incompréhension, et de signaler ces types de situations et d'y rémédier. Pour assurer l'efficacité de l'échange, les moyens de divers types sont utilisés, notamment les gestes et des signaux vocaux et non verbaux.

Les opérations discursives (OD) de reconstruction de sens qui sont à la disposition des participants dans le procès de communication exolingue, se situent sur l'axe « explicite vs. implicite ». Il est possible de distinguer A) les opérations explicitantes de reconstruction de sens et $\mathrm{B}$ ) les opérations implicitantes de reconstruction de sens. Parmi les opérations explicitantes de reconstruction de sens, la reformulation occupe une position dominante. Conçue de manière large, cette stratégie abrite une série de phénomènes relevant, pour la plupart, de l'ordre de la reprise modificatrice. La reformulation peut être intralinguistique ou interlinguistique. Dans ce dernier cas, elle est liée au transcodage (la traduction). Les opérations implicitantes de reconstruction de sens sont, dans une large mesure, basées sur l'expérience du locuteur, sur son niveau de compétences communicatives et sur sa capacité d'exploiter, de manière active, son répertoire langagier et métalangagier. Il s'agit de l'inférence, conçue en termes de la prédiction contextuelle et, plus généralement, de la reconstruction du

XLinguae, Volume 13 Issue 1, January 2020, ISSN 1337-8384, eISSN 2453-711X 
sens à partir du contexte linguistique. L'intercompréhension linguistique, faisant également partie des $\mathrm{OD}$ du type $\mathrm{B}$, renvoie à la reconnaissance des formes linguistiques au terme d'un travail de comparaison et d'association, et à la formulation et à la vérification des hypothèses portant sur les contenus sémantiques à identifier, en exploitant les connaissances sur les liens de parenté entre les différentes langues.

Les opérations discursives mentionnées ci-dessus peuvent être envisagées non seulement sous les perspectives «explicite vs implicite» mais également sous perspective de leur caractère intralinguistique ou interlinguistique. L'exemple de la reformulation montre la bi-valence de certaines opérations. De fait, la reformulation peut se situer dans les limites d'une langue; dans d'autres cas, elle les dépasse pour se coupler avec le transcodage (la traduction). En ce qui concerne la traduction, conçue comme une des opérations palliatives à des obstacles inhérents à la communication exolingue, elle sera étudiée sous le grand chapeau des opérations d'ordre reformulatif. Dans le cadre de notre recherche, les phénomènes liés au changement de code et au mélange de codes, au sens traditionnels des termes, reçoivent une attention à part (cf. aussi Rackova - Schmitt 2019 pour une analyse minutieuse des rapports particuliers d'interférence entre le français et le slovaque). Ils sont abordés sous perspectives distinctes. Pour le changement de code (l'alternance codique), la perspective est celle de l'examen des cas de la juxtaposition des segments en langues différentes. Pour le mélange des codes, également présent dans le corpus, la perspective adoptée est celle de l'examen des interlangues en oeuvre chez les locuteurs et l'examen des cas où les locuteurs décident de recourir à une lingua franca (cf. Veverkova - Gallayova 2015). L'intérêt de la recherche porte sur les spécificités du rôle communicatif de "pivot ». Il porte également sur la représentation des différentes opérations discursives dans la parole des participants à un échange exolingue (et sur une éventuelle prédominance de l'une d'entre elles), sur les modes de leur coexistence et sur les synergies ou conflicts éventuels entre elles. Il s'agit notamment de découvrir le rapport entre, d'un côté, la compréhension implicite en s'appuyant sur un répertoire linguistique plus ou moins vaste (déterminé chez chacun par le nombre des langues maîtrisées et les niveaux de leur maîtrise ; ainsi que par les relations systémiques, génétiques et typologiques entre ces langues et par le degré de la connaissance de ces relations) et, de l'autre côté, l'explicitation reformulatrice du sens grâce au travail médiateur des locuteurs-pivots. Ainsi, deux hypothèses sont formulées :

Hypothèse 1 : Les locuteurs «pivots » parlent plus que les locuteurs «non pivots ».

Hypothèse 2: Dans la parole des «pivots », l'opération de reformulation intralinguistique est majoritaire par rapport aux autres opérations discursives explicitantes et implicitantes (notamment à la traduction et à l'intercompréhension).

\begin{abstract}
Analyse des données
La présence des différentes stratégies a été observée dans l'enregistrement no. $1 \mathrm{du}$ corpus dont la durée totale est de 60 minutes et 49 secondes. La spécificité de cet enregistrement par rapport aux autres qui forment le corpus est la présence de 4 participants dont deux, par leurs répertoires langagiers, seraient candidats au rôle de pivot.

Parmi les quatre locuteurs, il y a 3 femmes (désignées comme $\mathrm{M}, \mathrm{K}, \mathrm{A}$ ) et 1 homme (C). Trois locuteurs (M, A, C) parlent français, l'une (K) ne le parle pas du tout. Parmi les locuteurs francophones, il y a deux Français natifs $(A, C)$ et une Slovaque $(M)$ avec un niveau de compétence en français de $\mathrm{C} 2$. Trois locuteurs $(\mathrm{M}, \mathrm{K}, \mathrm{C})$ parlent slovaque. Parmi ces locuteurs slovacophones, il y a deux Slovaques natives (M, K) et un Français dont le niveau de compétence en slovaque est $\mathrm{C} 1$. K est une Slovaque qui ne parle pas du tout français, A est une Française qui maîtrise le slovaque au niveau A1. C est un Français avec une solide maitrise non seulement du slovaque mais aussi du tchèque (au niveau C2), langue étroitement apparentée. La transcription fait état du
\end{abstract}


niveau verbal de l'événement communicatif, ainsi que les éléments paraverbaux et non verbaux choisis (prosodie, pauses, rires, indices d'allocution etc.).

L'enregistrement contient 1442 interventions des participants, dont M produit $478, \mathrm{~K}$ produit 330, A produit 379 et $\mathrm{C}$ en produit 255. Si on répartit l'enregistrement en trois phases, chacune de 20 minutes approximativement, correspondant grosso modo, successivement, à l'introduction, à la partie centrale et à la conclusion, on peut observer l'activité énonciatrice croissante ou décroissante des quatre locuteurs dans les phases différentes (Tableau 1).

\begin{tabular}{|r|r|r|r|r|}
\hline Locuteur & Phase 1 & Phase 2 & Phrase 3 & Total \\
\hline M & 238 & 104 & 136 & 478 \\
\hline K & 136 & 114 & 80 & 330 \\
\hline A & 162 & 114 & 103 & 379 \\
\hline C & 89 & 56 & 110 & 255 \\
\hline Total & $\mathbf{6 2 5}$ & $\mathbf{3 8 8}$ & $\mathbf{4 2 9}$ & $\mathbf{1 4 4 2}$ \\
\hline
\end{tabular}

\section{Tableau 1. La parole des participants en termes de nombres d'interventions.}

En évaluant les nombres d'interventions produites par les différents locuteurs, force est de constater que les locuteurs susceptibles d'agir comme pivots (dotés de répertoires langagiers plus universels que les autres interlocuteurs), donc les locuteurs $\mathrm{M}$ et $\mathrm{C}$, ne monopolisent pas nécessairement l'espace communicatif ni ne sont pas obligatoirement plus présents que les autres. Néanmoins, leur présence peut être plus saillante que celle des autres (le cas de la locutrice $\mathrm{M}$ ), notamment si le pivot locuteur natif de la langue principale de la communication, le slovaque dans notre cas, dans laquelle les participants étaient censées de communiquer en priorité - ressent une responsabilité accrûe du bon déroulement de l'échange. Le locuteur C intervient, dans les deux premières phases, moins que tous les autres. Ainsi, l'hypothèse $n$. 1 n'a pas éfé confirmée.

Deux vois d'accès ont été choisies pour aborder l'analyse relativement complexe du travail des pivots :

$\mathrm{R}$ : l'activité reformulatrice

- $\quad \mathrm{R}_{\mathrm{n}}$ - les reformulations intralinguistiques non modificatrices (les répétitions « fidèles »),

- $\mathrm{R}_{\mathrm{m}}$ - les reformulations intralinguistiques (les reprises entraînant une modification sémantique),

- $\quad \mathrm{R}_{\mathrm{tr}}-$ les reformulations interlinguistiques (les traductions).

$\mathrm{C}:$ la juxtaposition des codes linguistiques

- $\mathrm{C}_{\mathrm{aut}}-$ les emplois autonymiques,

- $\mathrm{C}_{\text {emp }}-$ les emprunts,

- $\mathrm{C}_{\mathrm{alt}}$ - les changements/les alternances codiques non traduisants (au sens traditionnel de juxtaposition de segments en langues différentes).

L'opération d'alternance de codes $\left(\mathrm{C}_{\mathrm{alt}}\right)$ cotoie, dans le corpus analysé, deux autres opérations: celle de mention autonymique $\left(\mathrm{C}_{\text {aut }}\right)$ et celle d'emprunt $\left(\mathrm{C}_{\mathrm{emp}}\right)$. Par rapport à $\mathrm{C}_{\text {alt }}$, ces deux $\mathrm{OD}$ occupent des positions nettement plus marginales et se distinguent par l'étendue plus limitée des segments concernés, ne portant que, pour la plupart, sur des mots ou sur des expressions isolées. Dans les échanges analysés, les termes isolés sont empruntés $\left(\mathrm{C}_{\mathrm{emp}}\right)$ à des langues étrangères pour faire référence à des

XLinguae, Volume 13 Issue 1, January 2020, ISSN 1337-8384, eISSN 2453-711X 
réalités étrangères correspondantes. C'est le cas, par exemple, de certains toponymes, dont Toulouse (exemple 1, ligne 12), mais aussi, ailleurs, Carcassone, Draguignan etc. :

(1)

$1 \mathrm{M}$ : a v košiciach si bola $\mathrm{v}$ alliance française

2 A: áno

$3 \mathrm{M}$ : dobre

4 A: pre môj štúdium

5 M: pre tvoje štúdium a čo si študovala

6 A: ako učit' po francúzsky

7 M: fle ako učit'

8 A: fle áno

9 M: francúžstinu ako cudzí jazyk

$10 \mathrm{~A}$ : áno toto

11 M: dobre tak potom kde si študovala vo francúzsku

12 A: v toulouse

D'autres noms propres tels les les noms des institutions (Alliance française - exemple (1), ligne 1 ci-dessus), les événements (Ça marche!) ou les activités/produits culturels sont également concernés. Cependant, une partie de noms propres fait l'objet de la traduction. Entre autres, cela concerne les toponymes couramment utilisés dans la langue principale de l'échange (le slovaque dans notre cas). Ainsi, les locuteurs dans l'enregistrement analysé font référence à Lotrinsko et non à la Lorraine ou parlent plus souvent de Francúzsko que de la France.

Le corpus contient des cas de mentions autonymiques. La mise en mention apparaît, le plus souvent, dans les interventions à caractère métalinguistique où les significations des mots sont discutées :

(2)

M: hej to sa povie draguer

$[\ldots]$

C: napríklad draguer le fond

Notons que, dès qu'il y a une introduction d'un élément en français au sein d'un énoncé construit en slovaque il peut s'agir également de la volonté de passer au jargon professionnel. Trois locuteurs sur quatre travaillent dans l'enseignement de langues étrangères, la quatrième est une chercheuse en sciences du langage. Ainsi, par exemple, l'oscillation entre učit' po francúzsky - fle - francúzština ako cudzi jazyk dans l'exemple 1 ci-dessus, ne représente pas une nécessité communicative, mais une valeur ajoutée liée au partage des mêmes outils relevant du domaine socioprofessionnel commun et, pour la plupart du groupe, des mêmes concepts interculturels (cf. sur le fonctionnement des notions relevant du domaine interculturel dans le contexte d'enseignement, cf. Birova 2018).

Le repérage, dans la parole des pivots, des marques tributaires des séries des phénomènes $\mathrm{R}$ et $\mathrm{C}$ définis ci-dessus a permis de mener une réflexion plus ciblée autour de quelques-uns de ces phénomènes, notamment autour de la reformulation, de la traduction et de l'alternance de codes. Notons d'emblée que toutes ces opérations consistent à introduire, au sein des interventions, des mots en langue différente de la langue de base (la langue dans laquelle l'énoncé a débuté ou dans laquelle a été construit l'énoncé précédent du pivot ou d'un de ses interlocuteurs). Dans notre analyse, les différences entre les phénomènes sont à expliquer sous les perspectives des oppositions «explicite vs implicite» et 
« intralinguistique vs interlinguistique ». Le principe de co-référence est également pris en compte. Ainsi, la traduction répond à un appel de clarification sémantique explicite. Le plus souvent, elle est sollicitée et/ou signalée (exemples 3a et 3b) et elle juxtapose, au sein du même énoncé ou dans l'intervalle des énoncés (presque) contigus, deux segments sémantiquement équivalents en deux langues différentes (dedina - village, commune dans l'exemple 3a; *nečasto - parfois niekedy - niekedi dans l'exemple 3b) :

(3a)

M: brusno dedina

A: ok

M: village plus petit

A: ok

K: a ako sa to povie po francúzsky

$\mathrm{M}$ : village

$\mathrm{K}$ : village

M: commune

(3b)

A: často mmh nečasto parfois ç'est

M: niekedy

A: niekedi

La reformulation, conçue comme une reprise exprimant un sens différent relativement à un énoncé-source antérieur (Martinot 2015 : 1), repose sur la co-présence, au sein de l'énoncé ou dans les énoncés distincts, des segments co-référentiels dans la même langue (aký šport - teda šport pasivne aktivne sous 4a ; tvoja rodina - tvoj otec mama sestra brat ainsi que už bola na slovensku - už prišli t'a pozriet'sem na slovensko sous $4 \mathrm{~b}$; klobásy - ne klobásy ktoré dáváš na tyč sous 4c) :

(4a) M: ozaj aký šport vás baví teda šport pasívne aktívne

(4b) M: tvoja rodina už bola na slovensku tvoj otec mama sestra brat už prišli t’a pozriet'sem na slovensko

(4c) C: to jsou klobásy ne klobásy ktoré dávaš na tyč ako který má dva zuby

Le changement de code se distingue de la traduction et de la reformulation en ce qu'il n'établit pas de rapport, déterminé par la présence d'un invariant sémantique, entre deux segments, relevant chacun d'une langue différente (exemples $5 \mathrm{a}, 5 \mathrm{~b}$ et $5 \mathrm{c}$ cidessous) mais, de manière balisée ou non, met en présence deux codes linguistiques au sein du même énoncé ou entre les énoncés contigus.

(5a) M: biarritz nejaké také pre- près de la frontière espagnole

(5b) M: voilà bon super vel'mi dobre rozumela

(5c) C: víš co to je ja viem (un segment en tchèque est suivi d'un segment en slovaque)

La parole des deux pivots contient un grand nombre de traces des opérations discursives dont ci-dessus. 


\begin{tabular}{|r|r|r|}
\hline OD & Pivot 1 (M) & Pivot 2 (C) \\
\hline Rn & $22,5 \%$ & $14,8 \%$ \\
\hline Rm & $38,3 \%$ & $11,1 \%$ \\
\hline Rtr & $17,5 \%$ & $4,9 \%$ \\
\hline Total série R & $\mathbf{7 8 , 3} \%$ & $\mathbf{3 0 , 8} \%$ \\
\hline Caut & $10,8 \%$ & $3,7 \%$ \\
\hline Cemp & $0,8 \%$ & $8,6 \%$ \\
\hline Calt & $10,0 \%$ & $55,6 \%$ \\
\hline Total série C & $\mathbf{2 1 , 7} \%$ & $\mathbf{6 7 , 9} \%$ \\
\hline
\end{tabular}

Tableau 2. La saillance des OD des séries A et B chez les pivots 1 (M) et 2 (C).

Sur le total de 238 interventions du pivot 1 (M) dans l'étape 1 de la rencontre, donc celle qui fera objet de la microanalyse des OD, il est possible de repérer 110 interventions marquées et 128 interventions non marquées $(50,4 \%)$, du point de vue de la présence des opérations discursives pertinentes. Le total de 120 marques des différentes opérations discursives exploitées ont été relevées au sein des interventions classées commes marquées. Parmi les interventions marqueés, celles qui contiennent des autonymes et celles qui contiennent des emprunts sont peu nombreuses ( 13 cas et 1 seul cas respectivement). Le pivot 1 adopte, le plus souvent, la stratégie de reformulation modifiante (46 occurrences, correspondant à 38,3\%).

De 89 interventions produites par le pivot 2 (C), le marquage est présent dans 64 $(71,9 \%)$ interventions. Les 25 interventions restantes sont non marquées. La stratégie prédominante adoptée par $\mathrm{C}$ est de parler tchèque, langue bien maîtrisée de lui et en même temps la plus proche possible, du point de vue génétique, du slovaque, langue principale de la rencontre. Une moitié des interventions est conçue - en entier ou en partie - en tchèque. Ces interventions sont classées comme des cas de changement de code. Le pivot 2 ne fait quasiment pas de traductions (4 cas seulement) ni ne reformule beaucoup ( 9 cas de reformulation modifiante). Le recours massif au tchèque trahit l'importance accordée par le pivot 2 à la compréhension implicite, basée sur la compétence d'intercompréhension des langues. De fait, le bilinguisme passif des locuteurs tchèques et des locuteurs slovaques a été prouvé par de nombreuses études empiriques. Un autre trait saillant de la parole de C, par lequel il se distingue de la locutrice $M$, est la présence relativement fréquente des marques de plusieurs stratégies différentes au sein de la même intervention. Ce phénomène est visible également dans la proportion entre le nombre de marques et le nombre d'intervention C. Quant à la reformulation modifiante, elle n'est pas saillante chez C non plus. Il privilégie décidément les reprises fidèles (les reformulations intralinguistiques non modifiantes).

Dans notre cas, il est possible d'observer 2 réalisations différentes du travail de «pivot». Le pivot 1 reformule beaucoup, répète, puis traduit. Le pivot 2 parle une langue pont, puis répète et reformule. Il est possible de conclure qu'il n'y a pas un seul modèle qui façonne le travail des pivots ; en toute probabilité, il y en a plusieurs, à dominantes pragmatiques différentes. L'hypothèse $n$. 2 ne se confirme que chez le pivot 1 , mais pas chez le pivot 2 . Ainsi, il nous est possible de constater que les deux hypothèses de travail ont été infirmées.

\section{En guise de conclusion}

Les contextes de communication où il y a contact de deux langues, imposent des exigences plus élevées aux locuteurs. S'il s'agit des langues qui ne sont pas en rapport de proximité génétique ou typologique mais qui, tout au contraire, ont un caractère typologique, une appartenance génétique et/ou aréale différente, l'étude des stratégies 
communicatives assurant l'efficacité de l'échange peut s'avérer enrichissante. L'appui actif sur l'intercompréhension linguistique entre les locuteurs, basée sur leurs connaissances des systèmes linguistiques et de fonctionnements des langues, notamment de la langue de l'interlocuteur, est probable. La co-construction du sens est tout de même très souvent accompagnée de reformulation. Cette activité a une importance cruciale pour le succès de la communication exolingue.

\section{Bibliographic references}

BANGE, P. 1992. À propos de la communication et de l'apprentissage de L2 (notamment dans ses formes institutionnelles). Acquisition et interaction en langue etrangcre, vol. 1, pp. 53-85.

BEHRENT, S. 2008. Le français comme langue de communication entre etudiants d'origines internationales. Synergies Europe, n. 3 (2008), pp. 71-81.

BERNIER, M. 2007. Obstacles à la communication dans une situation de communication exolingue en contexte de clavardage. In: Boissonneault, J. - Bernier, M. (eds.) : Actes de la 13e Journee Sciences et Savoirs. Sudbury : Acfas-Sudbury. pp. 201-229. ISBN 978-0-88667-071-9.

BIROVA, J. 2018. La verification empirique de mise en place des concepts de l'interculturel dans le contexte FLE. Berlin, Bern, Bruxelles, New York, Oxford, Warszawa, Wien : Peter Lang. ISBN 978-3-631-74302-7.

CAPPELLINI, M. 2017. La telecollaboration vue par la communication exolingue Pour un enrichissement mutuel de deux traditions de recherche. Alsic, vol. 20, n. 2 . DOI : $10.4000 /$ alsic.3128.

DAUSENDSCHON-GAY, U. 2003. Communication exolingue et Interlangue. Linx, n. 49 , pp. 41-50.

DE PIETRO, J.F. 1988. Vers une typologie des situations de contacts linguistiques. Langage et societe, n. 43, pp. 65-89.

HUDELOT, C. - VASSEUR, M.T. 1997. Peut-on se passer de la notion d'etayage pour rendre compte de l'elaboration langagicre en L1 \& L2 ?. CALaP 15, pp. 109135.

KERBRAT-ORECCHIONI, C.: L'enonciation. De la subjectivite dans le langage. Paris : A. Colin, 2002. ISBN 9782200262686

MARTINOT, C. 2015. La reformulation : de la construction du sens à la construction des apprentissages en langue et sur la langue. Corela 18 (2015), pp. 1-23. ISSN 16385748.

PLANTIN, Ch. - KERBRAT-ORECCHIONI, C. 1995. Le trilogue. Lyon : Presses universitaires de Lyon. ISBN 9782729705176

PORQUIER, R. 1979. Strategies de communication en langue non-maternelle. Travaux du Centre de Recherches Semiologiques de Neuchatel 33, pp. 39-52.

PORQUIER, R. 1984. Communication exolingue et apprentissage des langues. In: Py, B. (ed.): Acquisition d'une langue etrangcre III. Neuchatel : Centre de linguistique appliquee - Paris : Presses Universitaires de Vincennes.

PORQUIER, R. 2003. Deux repcres de recherche sur l'interaction exolingue. Linx, $n$. 49, pp. 51-62.

RACKOVA, L. - SCHMITT, F. 2019. Les interferences linguistiques du français sur le slovaque. L'exemple du systcme verbal. Paris : L'Harmattan. ISBN 978-2-34318000-7.

ROSEN, E. - REINHARDT, C. 2003. Les risques (limites) du metier... La dimension de l'expertise dans le continuum exolingue/endolingue. Linx, n. 49, pp. 91-108.

VEVERKOVA, D. - GALLAYOVA, Z. 2015. Anglicky jazyk ako nastroj vyucby Globalneho vzdelavania na Technickej univerzite vo Zvolene. In : Veverkova, D. Danihelova, Z. - Luptak, M. (eds.): Aplikovane jazyky v univerzitnom kontexte -

XLinguae, Volume 13 Issue 1, January 2020, ISSN 1337-8384, eISSN 2453-711X 
elektronicka podpora vzdelavania. Zvolen : Technicka univerzita vo Zvolene, pp. 3446. ISBN 978-80-228-2797-3.

Words: 5207

Characters: 34326 (19,07 standard pages)

doc. PhDr. Katarína Chovancová, PhD.

Département d'études romanes, Faculté des Lettres

Université Matej Bel de Banská Bystrica

Tajovského 51

97401 Banská Bystrica

Slovaquie

katarina.chovancova@umb.sk

Mgr. Carolina Haro Guerrero

Département des Lettres

U. F. R. Langues, lettres et communication

Université Rennes 2 - Haute Bretagne

Place du Recteur Henri Le Moal CS 24307

35043 Rennes cedex

France 